\title{
Needs and prospects for epidemiological tools in leprosy control
}

\author{
P FEENSTRA
}

\section{Introduction}

\subsection{MAGNITUDE OF THE LEPROSY PROBLEM}

The suffering of leprosy is caused by irreversible damage to the peripheral nerves, which leads to sensory loss, paralysis and loss of function of the hands, feet and eyes. The resulting deformities are the main cause of the social stigma attached to the disease. This stigma especially leads to the very serious psychological, social and economic consequences for leprosy patients as well as for their families. It should therefore be realized that the magnitude of the leprosy problem is insufficiently reflected by mere data on numbers of cases.

In September 19903.7 million leprosy cases were registered worldwide for chemotherapy. During 1990, 576,360 new cases were reported. (WHO) This information is obtained from routine health services reports from the individual countries and should therefore be treated with caution. It is, however, the best information available on the global leprosy problem.

\subsection{IMPACT OF MDT}

The introduction of the WHO recommended multidrug therapy (MDT) over the last 10 years was a revolutionary event in the history of leprosy control. MDT has proven to be effective, safe and acceptable to patients and health workers and can be implemented under a wide variety of conditions. Early relapses are rarely observed. The shortened duration and the effectiveness of the treatment improves patient compliance and motivates health workers. In most programmes the introduction of MDT is included in the intensive (re)training of the health workers, health education of patients and the community as well as improved supervision; this has resulted in a considerable upgrading of leprosy control activities. These positive effects of the implementation of MDT have brought about a growing confidence in the community concerning the possibilities of leprosy treatment. Many programmes have reported that this has resulted in increased early self-reporting of patients so that in more cases nerve damage is prevented.

Because of the short duration of MDT, patients are released from treatment much earlier than with dapsone monotherapy. This has, together with the initial 'clearing of the registers' during the introduction of MDT, resulted in a striking decline of the number of 
leprosy patients registered for treatment. An impressive example is the ALERT Leprosy Control Programme in Shoa Province, Ethiopia, where the number of patients registered for chemotherapy fell from 21,138 in 1982 to 2,636 in 1990 . On a global scale the number dropped from 5.4 million in 1985 to 3.7 million in 1990, although by that time only $55 \%$ of the cases were on MDT.

MDT has not only a higher efficacy than dapsone monotherapy, but as mentioned above, in many programmes patients are also diagnosed and treated at an earlier stage of the disease. This means that the period of infection is shortened. Therefore it is possible that the risk of infection will become lower in an area covered by an MDT programme than in a comparable area where the leprosy control programme relies on dapsone monotherapy. If this assumption is true and if it is further assumed that, in analogy with tuberculosis, there is a direct relation between the risk of infection and the incidence of disease, a decline (or an acceleration of an already existing declining trend) in the incidence of leprosy may be expected during the next 10 years in those areas where MDT has been widely and adequately implemented during the 1980 s.

Given the long incubation time of leprosy and the fact that in most programmes wide MDT coverage of cases has not yet, or only very recently been achieved, a clear idea of the impact of MDT on the incidence of leprosy is not yet available. Nevertheless, the incidence of leprosy (as reflected by case-detection figures) is clearly declining in a number of countries where MDT was introduced in the early 1980s and where there were well organized leprosy control programmes based on dapsone monotherapy many years before the introduction of MDT, e.g. Thailand. This decline in incidence, in addition to a possible initial effect of MDT, is mainly attributed to the intensive decades-long implementation of dapsone monotherapy. However, many other known factors, (e.g. vaccination with $\mathrm{BCG}$ ) and unknown factors (e.g. related to socio-economic improvement), may have played a role. Moreover, the decline in incidence is not observed in all countreis which have implemented MDT since the early 1980s and had a good monotherapy programme prior to the introduction of MDT.

\section{Elimination of leprosy}

The 44th World Health Assembly (1991) adopted as a resolution the goal of attaining global elimination of leprosy by the year 2000. 'Elimination' is defined as reaching a level of prevalence below 1 case per 10,000 population. The prevalence of leprosy (number of cases registered for treatment at a specified time) is equal to the product of the incidence (number of new cases detected during the year) and the average duration of treatment. Given the global number of cases annually detected (1989/1990: 576,360; case detection rate $=1.09$ per 10,000 population!), it may be expected that the global elimination goal can be achieved with the present MDT regimens, provided full coverage with MDT is established within the next few years and is maintained at the highest possible level. This expectation is justified if the duration of treatment does not exceed a period of 6-9 months for PB leprosy and of 24-36 months for MB leprosy. As the global MB:PB ratio among new cases is below 2:3, the prevalence will become more or less equivalent to the incidence. For individual countries, which at present have case-detection rates above 1 per 10,000 population, the target is likely to be achieved within 10 years only if the local $\mathrm{MB}: \mathrm{PB}$ ratio is very low and if full MDT coverage is established soon. 
However, by the end of 1990 only $55.7 \%$ of the 3.7 million leprosy patients registered for chemotherapy was on MDT. Far too many leprosy patients do not yet have access to the benefits of MDT, especially in the African Region, which with an MDT coverage of only $18.4 \%$ is lagging behind. Successful introduction of MDT so far has been achieved mainly in those countries or regions where the conditions are relatively 'easy' for the implementation of MDT: countries or regions with a good infrastructure, or with sufficient numbers of well-trained health workers, a good coverage with health services, or with a pre-existing, well-managed leprosy control programme based on dapsone monotherapy, adequate financial resources etc. It is obvious that major efforts are required for the achievement of full global MDT coverage.

\section{Integration of leprosy control}

At various occasions, including the 44th World Health Assembly, the WHO has urged the Member States to integrate leprosy control activities within the general health services. In many countries leprosy control programmes are already integrated or are in a process of transition from a specialized, vertical structure towards integration. The concept of the integration of leprosy control is gaining wide acceptance. To a great extent this is based on considerations of efficiency. With the declining number of registered patients vertical programmes are far too expensive. However, the basic justification for integration is the principle of equity. Integration means that leprosy control becomes the integral responsibility of permanent, community-based general health services, which provide the entire community, including leprosy patients, with comprehensive and continuous health care. This has clear advantages compared to the periodical and monopurpose services of vertical programmes, which in many situations reinforce the stigma attached to the disease. A prerequisite for integration is the existence of an adequately functioning general health services infrastructure. Although at the central and intermediate levels specialized services should be maintained within the integrated programme (for training, technical supervision, referral), the day-to-day patient management and recording, registration and reporting will become the task of general health staff.

\section{Needs for appropriate epidemiological tools}

\subsection{CHANGING NEEDS}

In 1983, when experience with MDT was very limited, a WHO Study Group on the Epidemiology of Leprosy in Relation to Control made a number of recommendations on the application of epidemiological tools for leprosy control (TRS 716, WHO, Geneva, 1985). The needs and relevance of various aspects of epidemiology of leprosy in relation to control have undergone significant changes since the Study Group made its recommendations.

In the light of:

the decreasing prevalence of leprosy as a result of the implementation of MDT; the WHO goal of reaching a global prevalence rate below 1 case per 10,000 population by the year 2000; and 
the widely accepted policy of implementing leprosy control activities as an integrated part of the general health services based on the primary health care approach,

there is a need to review these recommendations and to identify how optimal use should be made of epidemiological tools for the management of leprosy control. Such management tools should assist in the planning, implementation, monitoring and evaluation of leprosy control programmes and should by definition be relevant for decision-making. The management tools should preferably be based on epidemiological information which can be collected by general health staff under routine field conditions.

In this context three important subjects, which are strongly inter-related, are to be considered:

the assessment of the leprosy problem in terms of prevalence and incidence of leprosy and leprosy-related disabilities;

the prediction of future trends of this leprosy problem;

the basic epidemiological data for monitoring and evaluation of leprosy control programmes under routine field conditions.

\subsection{ASSESSMENT OF THE LEPROSY PROBLEM}

A reasonable knowledge of the magnitude of the leprosy problem in terms of prevalence and incidence of leprosy and leprosy-related disabilities is required for adequate planning, implementation and evaluation of leprosy control activities. At present the estimated case-load ranges from 7 to 15 million worldwide. This wide range demonstrates the lack of adequate tools to measure the magnitude of the leprosy problem. The issue is further complicated by the lack of a clear operational definition for a case of leprosy.

The only method available so far for obtaining reliable estimates is the (random sample) prevalence survey. Because of the low prevalence of leprosy and its tendency to occur in clusters, the sample size has to be very large. This makes the method an expensive and time-consuming affair, which is not appropriate for application in the vast majority of leprosy-endemic countries.

There is, therefore, a need for practical methods for the assessment of the leprosy problem which are adequate for the planning and evaluation of leprosy control programmes. In this context it should be carefully considered what degree of precision is required for the planning of leprosy control activities and whether the methodology is appropriate for application under very low prevalence conditions. The methods should preferably be based on available health services data, as it may be assumed that in general the information on registered cases reflects the magnitude of the leprosy problem in a country. However, different extrapolation factors will apply for different situations.

\subsection{PREDICTION OF FUTURE TRENDS}

Predictions of future trends are useful for long-term health planning and are important for obtaining commitment from governments, funding agencies and health authorities. Expectations regarding the future trend of the incidence and prevalence of leprosy and leprosy-related disabilities, as discussed in Section 1.2, are mainly based on extrapolation of the trends of reported numbers of cases on register and reported numbers of new cases. It may, however, be questioned whether these expectations are based on scientifically 
sound evidence. Not only are there many hazards involved in the extrapolation from routine health services data, but as there is also a lack of a standardized operational definition of a case, the reliability of these predictions is even more questionable. Moreover, the impact of MDT on the risk of infection is not known, as there are no tools available to measure infection. And even if infection could be measured, the relation between the risk of infection and the incidence of disease would still have to be identified.

There is, therefore, a need to identify or to develop scientifically sound epidemiological tools for the prediction of future trends, which can assist in the planning of leprosy control. Such tools may consist of a combination of various forecasting techniques. The issue also includes the exploration of the possible use as a management tool of epidemiological techniques, especially modelling, for the identification of optimal interventions for the reduction of the leprosy problem (e.g. surveillance of high risk groups, use of immunodiagnostic tests, chemoprophylaxis, alternative drug regimens, duration of treatment, vaccine) under varying epidemiological and operational conditions.

An important question, which has to be addressed here, is whether it will be feasible to develop tools which are of practical relevance for programme managers. What will be their limitations and how easy will they be to apply? Will they be precise enough for planning the elimination of leprosy in the individual countries?

\subsection{BASIC DATA REQUIREMENTS FOR THE EVALUATION OF LEPROSY CONTROL PROGRAMMES}

The basic strategy for leprosy control consists of early diagnosis and the provision of adequate chemotherapy ( $=$ a complete course of MDT) in order to:

cure the patients;

to interrupt transmission of infection, thereby reducing the incidence of disease; and to prevent leprosy-related disabilities.

Therefore the most relevant indicators for the monitoring and evaluation of leprosy control should reflect the programme effectiveness in terms of the proportion of cases on treatment among the real number of cases, the proportion of patients cured $(=$ completed treatment) among those who started MDT, the reduction of the incidence of leprosy and the reduction of the incidence of leprosy-related disabilities.

The indicators should be appropriate for application in low prevalence situations. They should preferably reflect directly what they are supposed to measure, e.g. proportion of patients completing treatment among those expected to complete treatment, but may also be proxy indicators, e.g. proportion of disabled patients among new cases as an indicator for the case detection performance.

Usually leprosy control programmes collect a wide variety of data. Many programmes have developed their own (sometimes computerized) information systems despite the availability of the OMSLEP system, which apparently does not fulfil everyone's requirements. Most leprosy control programmes do not make adequate use of the data, often obtained through complicated administrative procedures and a lot of field workers' and their supervisors' working time. Most of this information is not used for any decision making. 
In order to improve this situation it is necessary to identify the major issues involved in the evaluation of the operational performance and of the epidemiological impact of leprosy control programmes. The decision on what events have to be reported and which data have to be collected should be based on these issues.

The indicators suggested in the report of the WHO Study Group on the Epidemiology of Leprosy in Relation to Control (TRS 716) are far too many and too many of them are not linked to decision-making or are appropriate for routine application in integrated programmes.

Therefore, there is a need to identify those indicators, including data requirements, which are the most essential as tools for the evaluation of leprosy control programmes. Moreover a number of additional indicators, which are appropriate for optional use by more advanced programmes and a number of indicators, which can be applied for special epidemiological studies, may be identified.

Some suggestions were formulated during the WHO Consultation on Technical and Operational Aspects of Leprosy, Male, Maldives, June 1990.

In order to obtain complete and reliable data the purpose and the meaning of the data should be clear to those who have to collect them: the peripheral multipurpose health workers. This implies that the data should be relatively simple to collect and that the amount of data should be limited. The same is required for the methods for patient registration and reporting. An assessment of the experience gained with the OMSLEP recording and reporting system for leprosy system for leprosy patients is expected to be very useful for this purpose.

The method and application by general health staff of the reporting of patients according to treatment cohorts needs further exploration. A major advantage of this method is that it allows cohort analysis, not only regarding the completion of treatment (which reflects, as a direct indicator, much better the patient management performance than the indicators as suggested by the WHO Study Group (TRS 716)), but also regarding the incidence of reactions, disabilities, relapses, etc.

In view of the above, the definition of a case of leprosy should be clarified. Clear and standardized operational criteria are required in deciding whether a leprosy patient is in need of chemotherapy or not. Pref erably the criteria should be based on clinical evidence. In order to exclude persons with 'sub-clinical' leprosy from the case registers, it has been suggested that a time criterion for patients with single skin lesions should be added, as the majority of such lesions are self-healing and do not lead to potentially disabling nerve function impairment.

The issue of the duration of treatment has to be considered as well, especially in relation to those cases still showing signs of active disease at the time of completion of treatment. Authoritative and clear operational criteria should be identified for the definition of 'cure'.

\subsection{STATE-OF-THE-ART ON EPIDEMIOLOGY OF LEPROSY}

In order to address the questions mentioned above it is necessary to review first the Stateof-the-Art on epidemiology of leprosy, especially in relation to the three main issues of this meeting: the rapid assessment of the leprosy problem, the prediction of trends and the identification of evaluation tools. 
The most important gaps in existing knowledge have to be identified and, consequently, suggestions for research should be made.

The following questions have to be addressed:

Which knowledge on the epidemiology of leprosy has been acquired during the past decade, which saw such a revolutionary change in the approach to leprosy control?

Which are the most important risk factors for leprosy, leprosy reactions and leprosyrelated disabilities? Is it possible to identify special high-risk groups which, especially under low prevalence conditions, are in need of specific interventions, e.g. clinical and/or bacteriological surveillance, prophylactic treatment, vaccination, etc.?

What might be the implications of human immunodeficiency virus (HIV) infection for future trends in the epidemiology of leprosy?

Is there sufficient scientifically sound evidence to justify the optimistic expectations concerning the elimination of leprosy? Is it justified to rely on the extrapolation of recently observed trends?

Is it appropriate to attribute the observed changing leprosy trends, especially regarding the incidence, to the impact of leprosy control programmes or is it possible (and how) to identify the influence of other, perhaps more important factors such as BCG vaccination or factors related to socioeconomic conditions?

Is it, given the limited period of post-MDT surveillance, justified to expect that the relapse rate after MDT will remain as low as is observed during the first years after stopping chemotherapy? Can it be predicted what will happen with the leprosy trends if, for example, relapse rates will progressively increase 10 years after release from chemotherapy?

\section{Scope of the meeting}

The purpose of this meeting is to identify or to develop scientifically sound and practical management tools for leprosy control based on appropriate epidemiological information. The tools should be appropriate for application in integrated leprosy control programmes and under low prevalence conditions. The outcome of the meeting is expected to contribute significantly to the improvement of leprosy control through the rationalization of the use of epidemiological information for the planning, monitoring and evaluation of leprosy control programmes.

In order to achieve its purpose the meeting will first review the State-of-the-Art on the epidemiology of leprosy. After this review the specific needs for tools for the assessment of the leprosy problem and for the prediction of trends will be addressed. Finally the meeting will concentrate on the identification of the most appropriate data requirements for the monitoring and evaluation of leprosy control programmes.

Based on the identified needs for management tools and the required epidemiological information, as part of the group discussions on the four main issues we should attempt to identify the priorities for research which are relevant for the improvement or development of such tools. This involves questions such as:

Is our present knowledge of the epidemiology of leprosy adequate for the identification and development of appropriate methods for the rapid assessment of the leprosy problem, for the prediction of trends and for the management and evaluation of leprosy 
control programmes under varying operational conditions and, if not, which research is needed to develop such tools?

Which research, especially in the field of health systems research, is needed for the better application of the available epidemiological knowledge of leprosy? Is it possible to evaluate the effectiveness of leprosy control programmes with routinely collected health services data or is there a need for special studies? What degree of precision is required of such data?

Which other new tools for leprosy control, such as a vaccine, new drug regimens, immunodiagnostic tests, etc., can be expected or should be developed during the next 10 or 20 years? What will their additional contribution, and thus their relevance, be in terms of potential benefit, efficiency and feasibility of implementation?

Practical and operationally feasible management tools are important in order to enable countries to participate effectively in the global elimination strategy for leprosy. The tools must be relevant for decision making and should preferably be based on data which can be collected routinely by multipurpose health workers at the peripheral level of the general health services. The meeting should keep in mind continuously that its aim is the identification of practical tools. Therefore we should not deviate into issues which may be very interesting, but are only of academic interest. Again and again we should ask ourselves: why and for what do we need the information and is it really needed for the improvement of leprosy control? 